\title{
Reflets
}

Revue ontaroise d'intervention sociale et communautaire

\section{Entre la performance et la consommation}

\section{Louis Durand}

Volume 9, numéro 2, automne 2003

Travail et mieux-être

URI : https://id.erudit.org/iderudit/011089ar

DOI : https://doi.org/10.7202/011089ar

Aller au sommaire du numéro

Éditeur(s)

Reflets : Revue ontaroise d'intervention sociale et communautaire

ISSN

1203-4576 (imprimé)

1712-8498 (numérique)

Découvrir la revue

Citer ce document

Durand, L. (2003). Entre la performance et la consommation. Reflets, 9(2), 24-31. https://doi.org/10.7202/011089ar

Tous droits réservés (C) Reflets : Revue ontaroise d'intervention sociale et communautaire, 2002

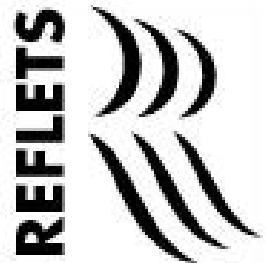

Ce document est protégé par la loi sur le droit d'auteur. L'utilisation des services d'Érudit (y compris la reproduction) est assujettie à sa politique d'utilisation que vous pouvez consulter en ligne.

https://apropos.erudit.org/fr/usagers/politique-dutilisation/ 


\section{Entre la performance et la consommation}

La majorité des personnes actives, quel que soit le type de travail qu'elles effectuent, entretient une relation ambiguë et contradictoire au travail qui varie en fonction de leurs conditions d'emploi. Pour caractériser cette relation, nous pourrions emprunter les mots de Danielle Linhart, qui disait: "le travail donne un sens à la vie et le travail empêche de vivre» ${ }^{1}$. Travail et mieux être, est-ce possible ou bien s'agit-il d'un rêve?

Pour ce numéro de Reflets, nous avons choisi de vous présenter, en lieu et place de l'entrevue conventionnelle, un court billet, une réflexion à voix haute sur les thèmes du travail, du mieux-être, de l'intégration au marché du travail. Ce billet a été rédigé par le professeur Louis Durand de l'École de commerce et d'administration de l'Université Laurentienne. Louis Durand est spécialiste en ressources humaines.

"La meilleure façon de tuer quelqu'un, c'est de l'empêcher de travailler» (Félix Leclerc).

«Et la pire? L'empêcher de consommer?» (L'auteur)

L. Durand : Le travail. Un mot générant beaucoup de discussions puisqu'il est au centre de nos vies quotidiennes. Larousse le définit de façon générale comme «l'activité de l'homme appliquée à la production, à la création, à l'entretien de quelque chose...» et plus spécifiquement comme «une activité régulière et rémunérée».Au départ, nous pouvons tous admettre ce besoin de faire, de progresser et d'apprendre par l'intermédiaire d'une activité, qu'elle soit physique ou mentale. Mais il y a plus, l'ensemble de ces activités sont regroupées sous le vocabulaire de postes et d'emplois rémunérés disponibles à l'intérieur d'un marché du travail, marché qui effectue en fait une distribution des ressources humaines visant à répondre 
aux multiples besoins de l'appareil productif au sein de nos sociétés. Cet appareil productif vise à combler les besoins des individus qui sont à la fois producteurs et consommateurs des biens et services ainsi produits. Par l'intermédiaire de ce cycle productif, décrit ici tout aussi succinctement que classiquement, nous travaillons, jour après jour, parfois avec grande motivation et parfois en questionnant notre raison d'être à l'intérieur de ce cercle. Nous nous sentons tous, un jour ou l'autre, comme cette petite souris qui court et fait tourner la roue en espérant atteindre le morceau de fromage.

Le processus de routinisation, qui nous assure une confiance minimale dans l'exécution de nos tâches quotidiennes, est de plus en plus difficile à gérer en période de changement continuel. L'insécurité qui en découle stimule notre réflexion et entraîne une remise en question de ce que nous faisons et de ce que nous sommes devenus au fil du temps. Que ce soit pour nos besoins psychologiques de réalisation personnelle ou tout simplement pour assurer la satisfaction de nos besoins physiques de base, le travail est en fait tout à la fois libérateur et usurpateur. Il est libérateur parce qu'il nous permet de participer à l'intérieur de la collectivité et de développer nos aptitudes et nos compétences. Il est usurpateur parce qu'il prend du temps. Le travail prend beaucoup de notre temps, mais le temps, c'est de l'argent et l'argent, ce n'est pas essentiel après tout, en autant que l'on en ait assez et assez, ça dépend de chaque personne. Ce lien fondamental entre le travail et l'argent a des conséquences qui peuvent s'avérer désastreuses avec le temps..., le temps que le travail nous a pris.

À chaque emploi est associé un statut social et le niveau de rémunération devient le principal facteur de catégorisation sociale. Une famille possédant deux automobiles et un garage double pour les loger, les automobiles je veux dire, se débrouille bien et peut être fière de son succès. A partir du moment où la consommation devient le principal élément de positionnement social, il va de soi que notre questionnement quant au sens du travail refait fréquemment surface. Par le travail, nous sommes ce que nous réalisons, et par la consommation, nous sommes ce que nous possédons. La consommation nous procure donc un outil 
pour galvaniser les progrès réalisés par la performance au travail, c'est le vaisseau d'or, mais pour le conserver, il faut continuer à performer. Il y a un prix à payer pour l'efficience sans limite, les psychologues et les psychiatres traitent de cette question... au besoin.

Une étude réalisée par Santé Canada en $2001^{2}$ trace un portrait pour le moins révélateur de l'évolution de la situation au cours des dix dernières années, pour ce qui concerne les heures consacrées au travail par les Canadiens. D'une part, alors que l'on pouvait trouver une personne sur dix qui consacrait plus de cinquante heures par semaine à son travail en 1991, ce taux est passé à une personne sur quatre en 2001. De plus, il apparaît que le travail supplémentaire non rémunéré dépasse largement le travail supplémentaire rémunéré. D'autre part, plusieurs études viennent confirmer que les symptômes de dépression voire d'épuisement professionnel (burnout) sont de plus en plus répandus au sein de la population. Que se passe-t-il exactement? Un simple regard sur les différents secteurs économiques amène toujours aux mêmes conclusions: mondialisation, compétition, déréglementation, innovations, changements et adaptation, et pourquoi pas, résistance et dépression. L'insécurité liée aux forts mouvements récessionnistes des années 1980 et 1990, qui s'accompagnaient bien évidemment d'une forte hausse du taux de chômage, a entraîné un changement majeur dans l'attitude au travail.Vous ne voulez pas faire d'heures supplémentaires?Vous avez peu de chance d'être promu et risquez même de perdre votre emploi puisque votre attitude ne correspond pas à la culture organisationnelle.

Derrière ce portrait supposément stimulant, un message est clair: plus vous êtes rapide et efficient, plus on vous donnera les ressources pour vous assurer une vie confortable... un jour, peutêtre..., si tout va bien et que vous êtes capable de maintenir le rythme. Plus encore, il m'est arrivé de remarquer à plusieurs reprises au cours des dernières années, que dans certains milieux, le travail et la fonction productive qui lui est associée, sont réifiés à des sommets insoupçonnés jusqu'à maintenant. Le succès professionnel prend des allures de rituel initiatique pour toute personne qui s'estime. Si on ne peut apprendre à s'apprécier qu'à 
travers le succès professionnel, l'enfance n'est donc plus qu'un mauvais moment à passer.

Dans une telle perspective, si vous ne travaillez pas, vous n'êtes que de peu de valeur. En effet, vous ne produisez rien, vous ne recevez aucun salaire et donc vous ne pouvez consommer que pour le strict minimum essentiel, puisque l'on ne vous donne droit qu'à quelques prestations et au plus bas des statuts sociaux. Conjoncture économique? Des excuses, bien sûr. Et que dire de la réforme de l'assurance-chômage, effectuée au cours des années 1990, qui fait en sorte que vous n'avez droit à aucun revenu si vous démissionnez de votre propre chef? Le principe premier de l'assurance-chômage est de permettre une certaine mobilité de la main-d'œuvre dans une perspective d'utilisation efficiente des ressources. Au Canada, au cours des vingt dernières années, le taux de chômage a toujours été nettement plus élevé que le taux de chômage naturel. Encore des excuses... la nature change elle aussi.

Les médias communiquent à toutes les semaines des histoires d'horreur: un jeune père de famille, mis en disponibilité, décide d'attaquer une banque; un homme dans la cinquantaine vient de perdre son emploi après trente ans de loyaux services, à cause d'une erreur d'inattention. Vous pouvez retourner à plusieurs reprises chez le garagiste ou le commerçant si vous n'êtes pas satisfait, mais vous, vous n'avez pas le droit de faire des erreurs ou de défaillir. La personne qui vous remplacera assurera le service aprèsvente. Vous voulez consommer? Il faut produire. Aux dernières nouvelles, l'économie mondiale est en surproduction et grâce à notre culture de l'efficience, nous surconsommons.

Ce cercle vicieux nous amène plus que jamais à développer notre réflexivité quant à notre rôle au sein de la société et quant à la valeur de notre contribution par l'intermédiaire du travail. Notre vie se divise en trois blocs distincts, le temps de travail, les temps de consommation et bien entendu le temps du roupillon. Est-ce que les énergies dépensées au travail ne peuvent se justifier psychologiquement que par notre capacité de consommer? C'est bel et bien l'approche qui est fondamentalement favorisée par le système économique et son appareil médiatique. Mais qu'en estil de chacun d'entre nous? Il y a encore aujourd'hui des gens tout 
à fait passionnés par leur travail et plus encore, des personnes qui, lasses de leur travail régulier et rémunéré, trouvent d'autres formes de travail pour faire, développer, créer et réaliser. Le travail est en effet la plus belle des opportunités pour être et vivre pleinement.

C'est l'industrialisation et la structuration des forces productives au sein de nos sociétés qui ont enclavé le travail dans des postes et des fonctions, des statuts et leur rémunération. Le vingtième siècle marquera l'histoire comme celui des organisations et qui dit organisations réfere à une panoplie de structures formelles et informelles, avec une distribution du pouvoir et une centralisation de la prise de décision. Au départ, l'individu s'accomplit dans le travail lorsqu'il peut agir sur toutes les étapes du processus et prendre les décisions qui lui semblent appropriées. Ce n'est plus le cas, sauf pour quelques privilégiés qui sont en haut de la pyramide..., ou à côté. Pour les autres, soit la majorité, la satisfaction se manifeste souvent durant les temps libres et par la consommation de tous les nouveaux produits assurant un meilleur confort.

Certes, ce début de millénaire semble teinté par un retour à une gestion plus saine des ressources humaines puisqu'il y a risque de pénurie de personnel compétent pour gérer l'économie du savoir. Pourtant, les impacts des années 1980 et 1990 demeurent omniprésents. Il est pour le moins extraordinaire que ce ne soit que depuis quelques années que nous parlions de la responsabilisation des employées et des employés, alors que nous avons fait des progrès majeurs en termes de qualité des ressources humaines par des investissements massifs dans nos systèmes d'éducation, et ce, depuis quarante ans. Pourquoi ne parle-t-on pas, ou si peu, de la responsabilisation des employeurs? L'incitation à l'exécution des heures supplémentaires non rémunérées a des impacts sociaux énormes, que l'on parle de santé mentale, de l'équilibre familial et de la qualité de vie des générations futures. Il faut cependant faire attention, les grandes entreprises sont de moins en moins attirées par les sociétés où les mesures sociales s'avèrent trop dispendieuses. La valeur de l'action sociale n'a que peu de poids face à la valeur de l'action ordinaire, toujours privilégiée. 
Notre relation au travail est donc profondément viciée, d'abord par notre crainte de perdre notre emploi et, ensuite, par notre dépendance au niveau de la consommation. Une forte proportion des Canadiens et des Canadiennes doit tout simplement aller travailler pour survivre et les considérations sur la valeur intrinsèque du travail s'avèrent superflues. Cependant, il semble que même l'atteinte d'une certaine sécurité dans l'emploi est rapidement remplacée par une forte incitation à la performance. Un regard furtif dans l'entourage nous permet de constater que ce n'est jamais assez: il faut agrandir la maison, choisir une automobile plus puissante et versatile, remplacer le petit écran de télévision par un système de cinéma à la maison et, pourquoi pas la voiturette électrique pour la pelouse..., sans oublier les vacances. Le bonheur à crédit nécessite une performance à tout prix.

Dans un tel contexte de performance, il apparait normal qu'il $y$ ait de plus en plus d'exclus et on pourrait pousser l'affirmation jusqu'à dire que les exclus apparaissent de plus en plus normaux. Les statistiques officielles ne sont qu'un portrait partiel et partial de la réalité. L'État est de plus en plus sévère pour les sans emploi: l'accès à l'assurance chômage maintenant rebaptisé sous le terme plus moderne d'assurance-emploi, est beaucoup plus difficile. On pourrait mentionner également que le nombre de travailleurs autonomes a doublé au Canada au cours des dix dernières années, peut-être par choix bien sûr, mais également par obligation. Quant à l'intégration sociale des immigrants, une étude révèle que c'est d'abord l'intégration au travail qui présente le plus de difficultés et ce, dans $70 \%$ des cas $^{3}$. Plusieurs d'entre eux doivent laisser de côté la formation reçue dans leur pays d'origine, pour aller alimenter la banque de travailleurs au salaire minimum. Le problème de la transférabilité des compétences entre pays nous laisse croire que la mondialisation est un phénomène conçu exclusivement par et pour les employeurs occidentaux.

Les lieux de travail ont toujours été des endroits privilégiés pour l'intégration des personnes au sein de la société. C'est souvent au travail que l'on rencontre la personne qui partagera éventuellement notre vie; c'est également le lieu où se développent des amitiés et des relations de confiance qui contribueront au 
développement de notre carrière. On peut cependant sérieusement questionner la qualité actuelle du marché du travail canadien pour assurer l'intégration sociale des immigrants et de tous les exclus de notre société. Si les personnes qui possèdent actuellement un emploi vivent déjà dans l'insécurité de le perdre, consacrent de nombreuses heures supplémentaires non rémunérées afin de le conserver et, dans une proportion notable, vivent soit des problèmes de santé mentale dûs au stress ou tout simplement des déséquilibres de plus en plus importants entre la vie au travail et la vie personnelle, il semble que les lieux de travail soient plus favorables à la désintégration.

Vers la fin des années 1970 et au cours des années 1980, on parlait d'une civilisation du loisir avec des semaines de travail beaucoup plus courtes et de nombreuses heures libres pour s'adonner à nos activités préférées. Les progrès technologiques devaient nous permettre d'atteindre une plus grande efficience et ainsi de consacrer moins d'heures au travail. Les années 1980 et 1990, avec leurs successions de récessions, nous ont cruellement fait réaliser que la souris court toujours et plus vite qu'avant, mais que le fromage semble s'éloigner davantage. L'accélération de la rationalisation des processus de production par le recours aux innovations technologiques successives a certes permis d'atteindre des sommets d'efficience, mais plutôt que de diminuer la pression exercée sur les personnes, elle a au contraire contribué aux problèmes de santé mentale que l'on connaît actuellement. Pourquoi? Parce que dans un marché compétitif, l'atteinte d'une plus grande efficience augmente naturellement la pression pour l'atteinte d'une efficience toujours plus grande. L'économie mondiale présente une surcapacité de production. Plus cyniquement, peut-être que si nous étions encore plus efficients, on pourrait sans aucun doute atteindre l'équilibre. On ne sait jamais...

Des solutions? Les manifestations pacifiques? Le retour à la terre? Non. D'abord, il faut une prise de conscience individuelle. Ensuite, il faut qu'il y ait partage de nos décisions avec notre collectivité immédiate, soit les collègues de travail. C'est d'abord et avant tout un choix personnel. Nous pouvons tous décider de redonner au travail la place qui lui revient dans notre vie: soit un 
tiers du temps. Il y a cependant un prix à payer: oublier une éventuelle promotion, peut-être changer d'emploi, conserver la maison, l'automobile et l'écran de télévision dans leur état actuel, le plus longtemps possible. Nous pouvons tous dire «Non» à la poursuite effrénée de l'efficience et à la pression exercée par l'employeur, mais il faut accepter d'en payer le prix. Lorsque cette prise de conscience deviendra collective, l'État devra légiférer et assurer pour ceux qui le veulent, les semaines de travail de quatre jours, les horaires variables, l'accès aux services de garde, les congés parentaux et tous les autres services qui permettraient une meilleure qualité de vie. Le prix à payer sera un niveau de vie plus bas pour une meilleure vie à tous les niveaux, exception faite de tous ces biens que nous voulions éventuellement remplacer. Refuser les heures supplémentaires n'est pas un signe de paresse mais bien de sagesse. Il n'est point question ici d'une meilleure répartition de la richesse, même si celle-ci serait favorisée par de telles mesures, mais bien d'une définition un peu moins barbare, voire matérielle de celle-ci. La richesse ne peut se résumer au travail et à la consommation, nous ne serions alors qu'une vile courroie de transmission. Une fois cette réflexion personnelle partagée et véhiculée auprès des générations montantes, peutêtre pourrons-nous alors recommencer à parler des milieux de travail comme des lieux favorables à l'intégration sociale.

\section{Notes bibliographiques}

1. Linhart, D. (1984). cité dans Marc Lesage, Les vagabonds du rêve, Montréal, Les éditions du Boréal Express, p. 39.

2. Higgins, C. et L. Duxbury (2002). Enquête nationale sur le conflit entre le travail et la vie personnelle (2001), Rapport 1. Santé Canada.

3. Statistique Canada (2003). «Enquête longitudinale auprès des immigrants du Canada», Le Quotidien, septembre 2003. 